\title{
The role of HDAC2 in chromatin remodelling and response to chemotherapy in ovarian cancer
}

\author{
Rui Huang ${ }^{1}$, Simon P Langdon ${ }^{1}$, Matthew Tse ${ }^{1}$, Peter Mullen ${ }^{2}$, In Hwa Um², Dana \\ Faratian $^{1}$, David J Harrison² \\ ${ }^{1}$ Division of Pathology, Institute of Genetics and Molecular Medicine, University of Edinburgh, Edinburgh EH4 2XU, UK \\ ${ }^{2}$ School of Medicine, University of St Andrews, St Andrews, Fife, KY16 9TF, UK. \\ Correspondence to: Simon Langdon, e-mail: simon.langdon@ed.ac.uk
}

Keywords: HDAC2, chromatin, platinum, ovarian cancer

Received: September 08, $2015 \quad$ Accepted: November 26, 2015

Published: December 14, 2015

\section{ABSTRACT}

Chromatin undergoes structural changes in response to extracellular and environmental signals. We observed changes in nuclear morphology in cancer tissue biopsied after chemotherapy and hypothesised that these DNA damage-induced changes are mediated by histone deacetylases (HDACs). Nuclear morphological changes in cell lines (PE01 and PE04 models) and a xenograft model (OV1002) were measured in response to platinum chemotherapy by image analysis of nuclear texture. HDAC2 expression increased in PEO1 cells treated with cisplatin at 24h, which was accompanied by increased expression of heterochromatin protein 1 (HP1). HDAC2 and HP1 expression were also increased after carboplatin treatment in the OV1002 carboplatin-sensitive xenograft model but not in the insensitive HOX424 model. Expression of DNA damage response pathways ( $P B R C A 1$, YH2AX, PATM, pATR) showed time-dependent changes after cisplatin treatment. HDAC2 knockdown by siRNA reduced HP1 expression, induced DNA double strand breaks (DSB) measured by YH2AX, and interfered with the activation of DNA damage response induced by cisplatin. Furthermore, HDAC2 depletion affected yH2AX foci formation, cell cycle distribution, and apoptosis triggered by cisplatin, and was additive to the inhibitory effect of cisplatin in cell lines. By inhibiting expression of HDAC2, reversible alterations in chromatin patterns during cisplatin treatment were observed. These results demonstrate quantifiable alterations in nuclear morphology after chemotherapy, and implicate HDAC2 in higher order chromatin changes and cellular DNA damage responses in ovarian cancer cells in vitro and in vivo.

\section{INTRODUCTION}

Chromatin structure is dynamic, and changes occur in response to extracellular and environmental signals [1]. Histone tail acetylation is an important chromatin modification that alters DNA accessibility to regulating enzymes by transforming chromatin from a compact to relaxed structure that is permissive of gene expression [2, 3]. The balance between histone acetyltransferase (HAT) and histone deacetylase (HDAC) activities ultimately determines acetylation status [4]. Histone acetylation is involved with cellular differentiation, mitosis and meiosis, DNA transcriptional regulation, DNA damage, DNA replication, and circadian rhythms $[5,6,7,8]$.
Mammalian HDACs are grouped into four classes based on structural homology, enzymatic activity, and cellular localisation $[9,10,11,12]$. Class I HDACs (HDAC 1, 2, 3, and 8) are mainly nuclear, and they interact with histones and other proteins [13, 14], while class II HDACs (HDAC 4, 5, 6, 7, 9, and 10) are tissuespecific and can be both nuclear and cytoplasmic [13]; the majority of HDAC inhibitors inhibit both class I and class II enzymes [15, 13]. Class III HDACs, namely sirtuins (SIRTs1 - 7; silent information regulators), are unresponsive to most HDAC inhibitors but require the cofactor NAD+. Finally, the class IV HDAC, HDAC11, is expressed in the nucleus and shares homology with class I and class II HDACs $[15,16]$. 
HAT and HDAC activity can be altered by mutation, overexpression, or translocation, disrupting the acetylation-deacetylation balance and consequently contributing to cancer hallmarks; these epigenetic changes have been observed in leukaemia and prostate, breast, colorectal, and ovarian cancers [17, 13]. Acetylation changes are thought to participate in carcinogenesis by silencing tumor suppressor gene promoters, such as p21, [18, 19], activation of repressed genes, or abnormal recruitment of HATs or HDACs [13].

The heterochromatin protein 1 (HP1) protein family plays various roles in establishing and maintaining heterochromatin (tightly-packed DNA) structure, thereby repressing transcription [20]. HP1 overexpression can cause global gene repression and chromatin condensation $[21,22]$. The three human HP1 isoforms, HP1 $\alpha, \mathrm{HP} 1 \beta$, and HP1 $\gamma$, share functions and localise to chromatin with incomplete overlap [23], differentially localising to centric heterochromatin, telomeres, and specific euchromatic sites [24]. Alterations in HP protein expression have been identified in some cancers including ovarian [25], breast [26], and colorectal cancer [27].

We have observed changes in nuclear structure in clinical samples of cancer tissue after treatment with chemotherapy and radiotherapy. Since structure dictates gene expression and, therefore, function, we sought to investigate this phenomenon to better understand therapeutic responses. We hypothesised that nuclear morphological changes in cancer in response to DNA damage are mediated by HDACs and are associated with changes in HP1 protein expression and/or nuclear distribution. Initial studies indicated changes in expression of HDAC2, therefore, we explored whether HDAC2 mediated response to injury and might act as a resistance factor to DNA-damaging therapy.

\section{RESULTS}

\section{Nuclear structure changes after chemotherapy or radiotherapy in ovarian cancer cells}

Preliminary observations by light microscopy suggested that nuclear morphology was different in clinical tumors after treatment with chemotherapy and radiotherapy (Figure 1). To explore this phenomenon further, chromatin patterns were quantified by nuclear texture image analysis in an ovarian cancer cell line model. Texture features were regarded as positively or negatively associated with chromatin patterns (homogeneity, heterogeneity, and contrast) as previously described [28, 29]. Five parameters associated with texture were obtained: angular second moment (ASM), correlation, entropy, inverse different moment (IDM) and contrast. ASM is a strong measure of uniformity or smoothness associated with overall homogeneity of chromatin patterns. Correlation calculates the grey-level linear dependency of the image and correlates negatively with the heterogeneity of chromatin patterns. Entropy measures pattern disorder and is negatively correlated with homogeneity. Inverse different moment (IDM) measures the local variability and intensity of a region of interest (ROI) and is affected by image homogeneity, with nonhomogeneous areas normally resulting in low IDM values; thus, it is described as the 'contrast' of chromatin patterns.

PE01 ovarian cancer cells [30] grown on coverslips were treated with cisplatin or ionising radiation and incubated for $0 \mathrm{~h}, 6 \mathrm{~h}, 12 \mathrm{~h}$ and $24 \mathrm{~h}$. After $24 \mathrm{~h}$ treatment with $6 \mu \mathrm{M}$ cisplatin or 6 Gy radiation, all five image texture parameters measured changed compared to untreated controls; observations were similar for both cisplatin and radiation. ASM, correlation, and IDM

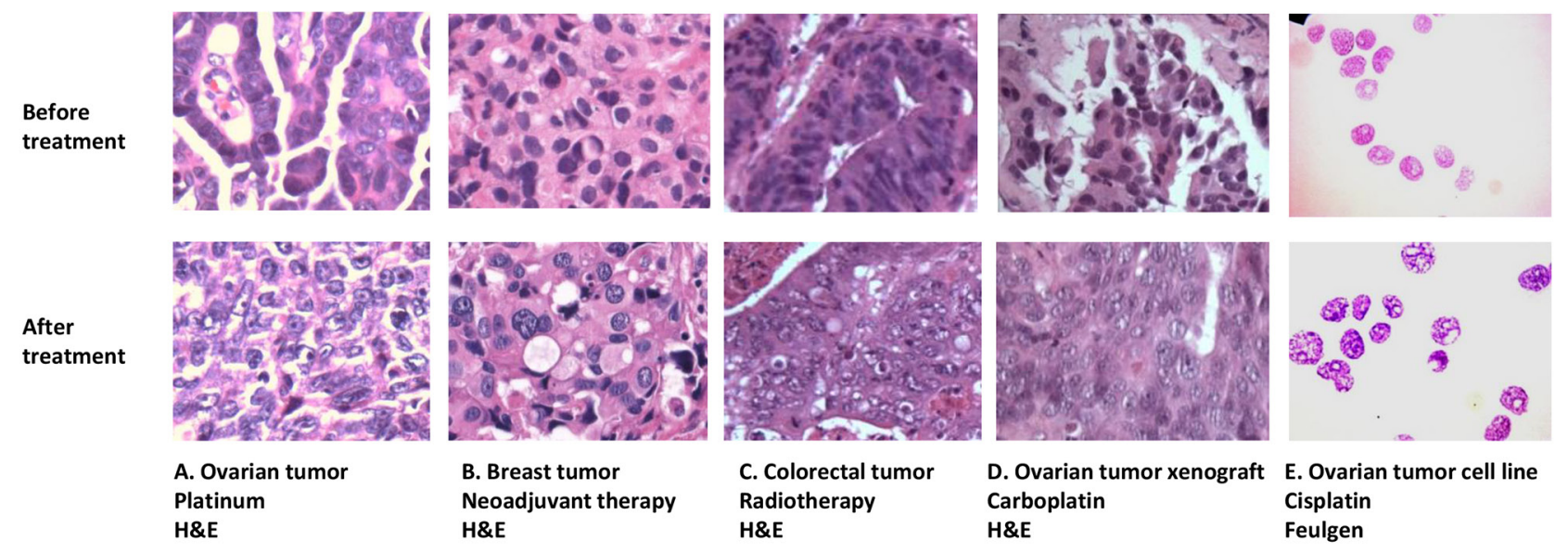

Figure 1. Nuclear morphology changes in different clinical and experimental settings. Similar nuclear texture changes occur in: A. ovarian tumors after chemotherapy; B. breast tumors after neoadjuvant therapy; C. colorectal tumors after radiotherapy; D. ovarian tumor xenografts after carboplatin treatment; and E. the ovarian cancer cell line PEO1 after cisplatin treatment. A-D are formalin-fixed, paraffin embedded sections cut from tumor samples and stained with H\&E. E shows PEO1 cell cytospins using the Feulgen nuclear stain. 
decreased after cisplatin/radiation treatment by $20 \% / 23 \%$, $25 \% / 49 \%$, and $15 \% / 11 \%$, respectively, while entropy and contrast increased by $6 \% / 8 \%$ and $40 \% / 120 \%$. The heterogeneity and contrast of chromatin increased and the homogeneity decreased in cell nuclei after DNA damageinducing treatment with cisplatin and radiation, consistent with the observations made by light microscopy.

\section{Measurement of nuclear texture changes in response to carboplatin in vivo}

We next sought to establish whether similar nuclear changes occur in vivo using a platinum-sensitive OV1002 patient-derived ovarian cancer xenograft model [31]. Carboplatin, a cisplatin analogue, was used as this drug is commonly used clinically. After a single treatment with carboplatin, ovarian cancer xenografts were collected on days $0,1,2,4,7$, and 14. Haematoxylin and eosin (H\&E) staining and light microscopy indicated similar morphological changes to those seen in vitro (Figure 1). Untreated tumors tended to have strongly stained and homogeneous nuclei, while nuclei after carboplatin treatment had more lightly stained nuclei and greater heterogeneity (Figure 2B). When nuclear texture was analysed by image analysis, texture parameters were different in samples after carboplatin treatment compared

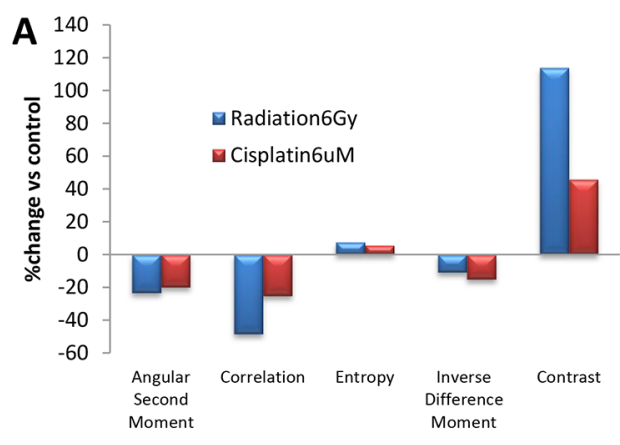

B
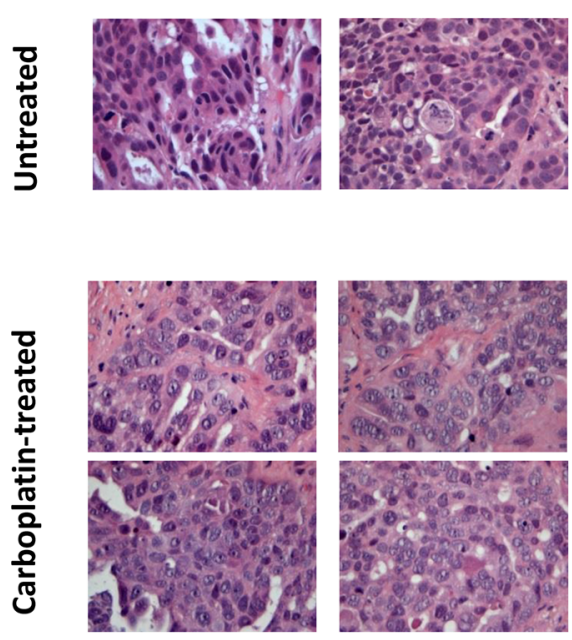

C

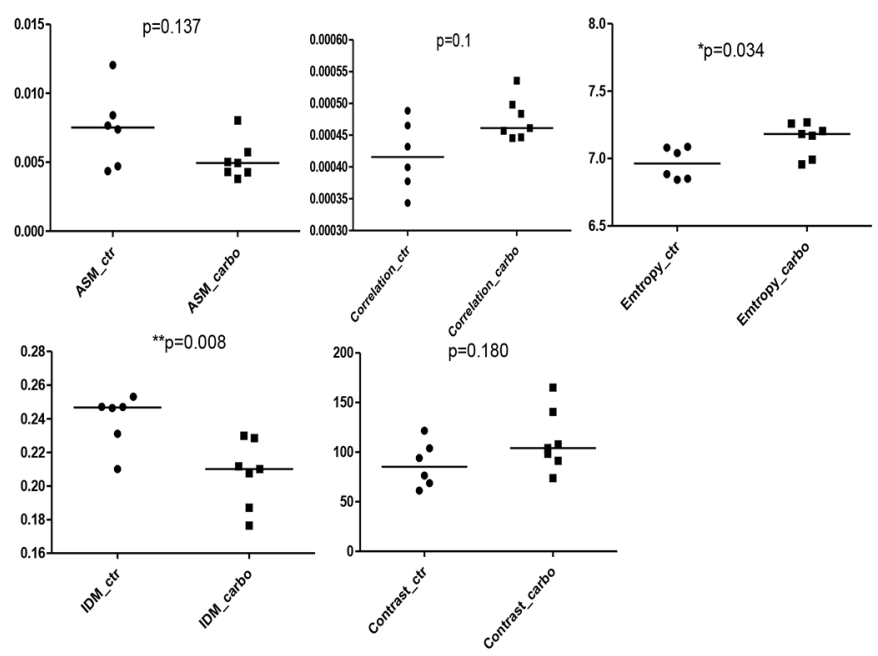

D

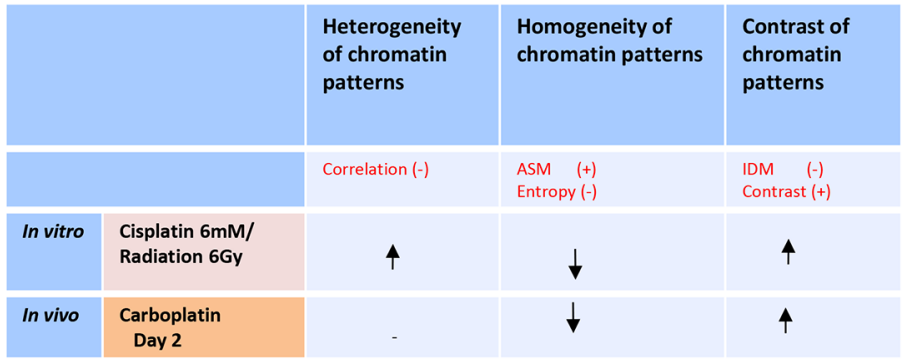

Figure 2. Alterations in nuclear texture in vitro and in vivo after treatment with platinum drugs or radiotherapy. A. Changes in nuclear texture in PEO1 cells after irradiation or cisplatin treatment. PEO1 cells were grown on coverslips and treated with ionising radiation (6Gy) or cisplatin $(6 \mathrm{uM})$ for $24 \mathrm{~h}$, and nuclei were stained with DAPI for visualisation using a fluorescence microscope. At least 100 nuclei were included in each experiment. Nuclear texture was analysed by measuring five texture parameters (angular second moment, correlation, entropy, inverse different moment, and contrast) using Image J software. Data are presented as the average change (\%) in the treated group for each parameter over the control group. B. Representative images from H\&E-stained OV1002 ovarian tumor samples either untreated or after carboplatin $(50 \mathrm{mg} / \mathrm{kg})$ treatment in vivo. H\&E stained images were acquired under 40x magnification. C. Nuclear texture parameter analysis in xenografts with and without carboplatin treatment on Day 2. Data for each spot represents the average value of each single sample with the number of nuclei analysed per sample ranging from 16 to 213 (average 103). Mann-Whitney U test (2-tailed); ${ }^{*} \mathrm{P}<0.05,{ }^{* *} \mathrm{P}<0.01$ D. Changes in chromatin patterns in PEO1 cells after cisplatin (6uM) or radiation (6Gy) treatment for 24h, and in OV1002 xenografts in vivo after carboplatin treatment, measured by parameters describing nuclear texture using Image J. The (+) and (-) represent positive and negative correlations with each type of chromatin pattern, respectively, and the arrows indicate the direction of change for each pattern. 
to controls, with the most significant effects of singledose carboplatin typically seen two days after treatment (entropy and IDM p=0.034 and 0.008, respectively; Figure 2C), indicating that chromatin pattern changes occur after platinum treatment in vivo and in vitro (Figure 2D).

\section{HDACs are differentially expressed in platinum- resistant cell lines}

It has previously been shown that HDAC1, HDAC3, and HDAC4 might be associated with resistance to chemotherapy and poor prognosis in cancer patients [32 - 34]. To investigate whether HDACs are involved in DNA damage-based treatment, we measured protein expression of HDAC class I (HDAC1, 2, 3, and 8) and
IIA (HDAC4) members in PEO1 and PEO4 cells 24h after cisplatin treatment. The PE04 cell line was derived from the same patient as the PE01 cell line but after resistance had developed [30]. Since changes in nuclear texture after cisplatin treatment were most pronounced at $24 \mathrm{~h}$, we speculated that HDACs would similarly show maximal changes at this time point. HDAC2 expression was increased approximately 1.5 fold in cisplatin-treated PEO1 cells, but not in PE04 cells, compared to controls at $24 \mathrm{~h}$. Expression of HDACs 1, 3, 4 and 8 were unchanged in both cell lines after $24 \mathrm{~h}$ (data not shown). This suggests that HDAC2 might be a cisplatin response biomarker in vitro, at least in sensitive cells. HP1 heterochromatin isoforms were also measured after cisplatin treatment (Figure 3A). Two HP1 isoforms (HP1 $\alpha$ and HP1 $\beta$ ) increased by about



B.
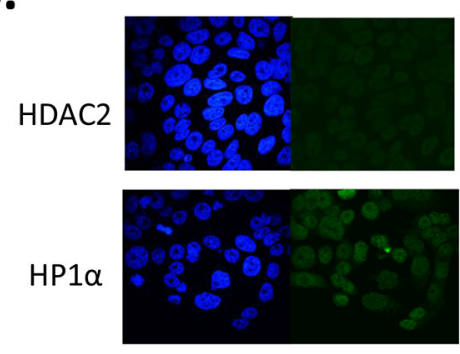

HP1ß



HP1ץ

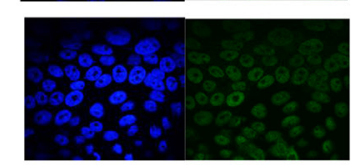

PEO4

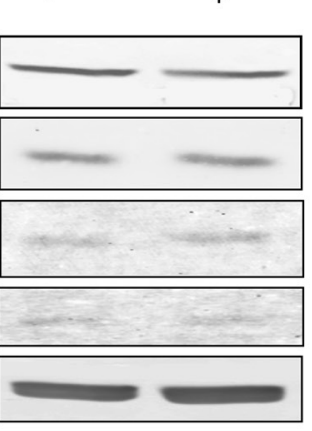

$6 h$
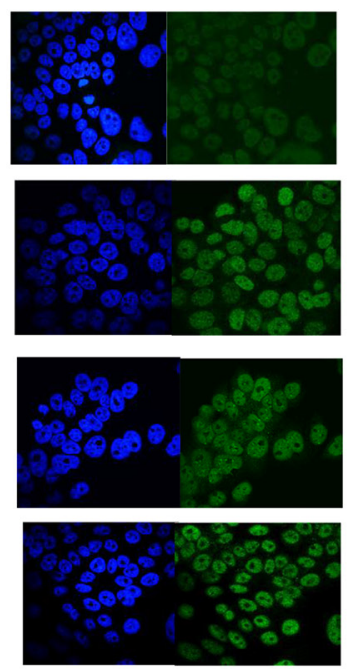

C.

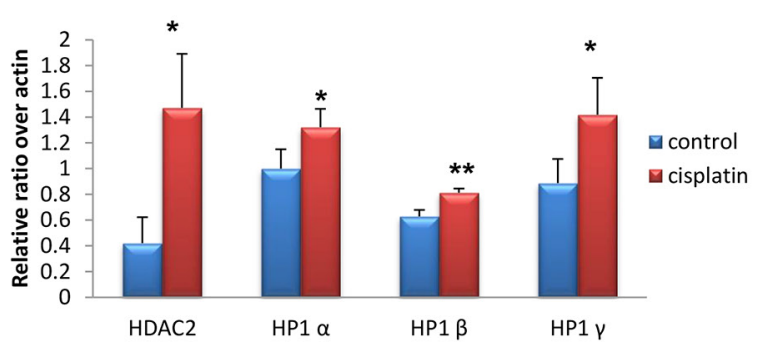

24h
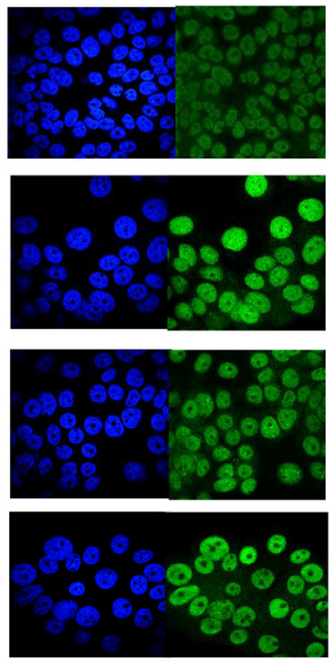

Target

DAPI

Figure 3. Expression of HDAC2 and heterochromatin proteins after cisplatin incubation. A. Western blots for HDAC2, HP1 $\alpha, \mathrm{HP} 1 \beta$, and HP1 $\gamma$ in PEO1 and PEO4 cells with or without cisplatin treatment (6 uM, 24h). Membranes were probed with the indicated antibodies, and tubulin was used as a loading control. B. HDAC2 and HP1 protein expression detected by immunofluorescence. Cells were seeded on cover slips and fixed as described in the Materials and Methods in PEO1 cells after cisplatin treatment for 6h and 24h. Alexa488 (green channel) and DAPI (blue channel) were used to stain target proteins and the nuclei, respectively. Images were taken using a confocal microscope. C. Expression of HDAC2, HP1 $\alpha$, HP1 $\beta$, and HP1 $\gamma$ mRNA in PEO1 cells measured by RT-PCR as described in the Materials and Methods. Relative expression of the target gene was calculated as the average $\Delta \mathrm{Ct}$ and normalized to that of the housekeeping gene $\beta$-actin. Results are as presented as mean $\pm \mathrm{SD}$ from biological triplicates. ${ }^{*} \mathrm{p}<0.05,{ }^{*} \mathrm{p}<0.01$ (Student's t-test). 
$30 \%$ and $70 \%$, respectively, but only in PEO1 cells after 24 $\mathrm{h}$ cisplatin treatment, while HP1 $\gamma$ protein remained static.

To visualise and confirm these observations, immunofluorescence (IF) was performed on PEO1 and PEO4 cells with or without cisplatin treatment using antibodies targeting HDAC2, HP1 $\alpha, \mathrm{HP} 1 \beta$, and HP1 $\gamma$ (Figure 3B). As expected, expression of HDAC2 increased $24 \mathrm{~h}$ after cisplatin treatment, and HP1 proteins gradually increased over $24 \mathrm{~h}$ of cisplatin treatment in PEO1 cells (Figure 3B). Again no changes were observed in PEO4 cells (data not shown). The IF images confirmed nuclear localisation of these targets.

Since HDAC2 and HP1 protein expression changed after cisplatin treatment in PEO1 cells, we next measured mRNA expression. Transcription of $H D A C 2, H P 1 \alpha, H P 1 \beta$, and $H P I \gamma$ were all significantly elevated by cisplatin treatment $(\mathrm{p}<0.05)$ after $24 \mathrm{~h}$ in PEO1 cells (Figure 3C), mirroring the protein expression changes; however, mRNA levels remained unchanged in PEO4 cells (data not shown). The changes in HP1 expression suggest that the amount of heterochromatin increases after DNA-damaging treatment.

\section{Profiling expression of HDAC and HP1 proteins in ovarian cancer xenograft models}

We then examined HDAC and HP1 expression in the platinum-sensitive OV1002 and platinum-resistant HOX424 xenograft models [31]. HDAC2 and all three
HP1 proteins were significantly increased in the sensitive model (OV1002) after carboplatin treatment, with the most significant changes $(\mathrm{p}<0.05)$ observed on day 7 (Figure 4$)$. In the HOX424 model, expression of these proteins was similar between control and treated groups.

\section{Time-dependent cellular DNA damage response induced by cisplatin in ovarian cancer cells}

We next investigated several DNA damage response (DDR) pathway members ( $\gamma \mathrm{H} 2 \mathrm{AX}, \mathrm{pBRCA} 1, \mathrm{ATM}$, pATM, ATR, and pATR) by western blotting (Figure 5). As expected, the DNA damage response proteins pBRCA1, $\gamma \mathrm{H} 2 \mathrm{AX}$, pATM, and pATR participated in the response to cisplatin and were upregulated after treatment. pBRCA1 expression increased after $24 \mathrm{~h}$ of cisplatin treatment in PEO1 cells, which persisted to $96 \mathrm{~h}$, while $\gamma \mathrm{H} 2 \mathrm{AX}$, pATM, and pATR increases occurred slightly later from $48 \mathrm{~h}$. ATM and ATR protein expression remained stable except for ATM reductions at $96 \mathrm{~h}$.

\section{Expression profiling of other HDAC family members, heterochromatin proteins, and DNA damage response proteins under $\mathrm{HDAC} 2$ suppression}

Given that HDAC2 expression showed the most pronounced changes in response to cisplatin, we examined

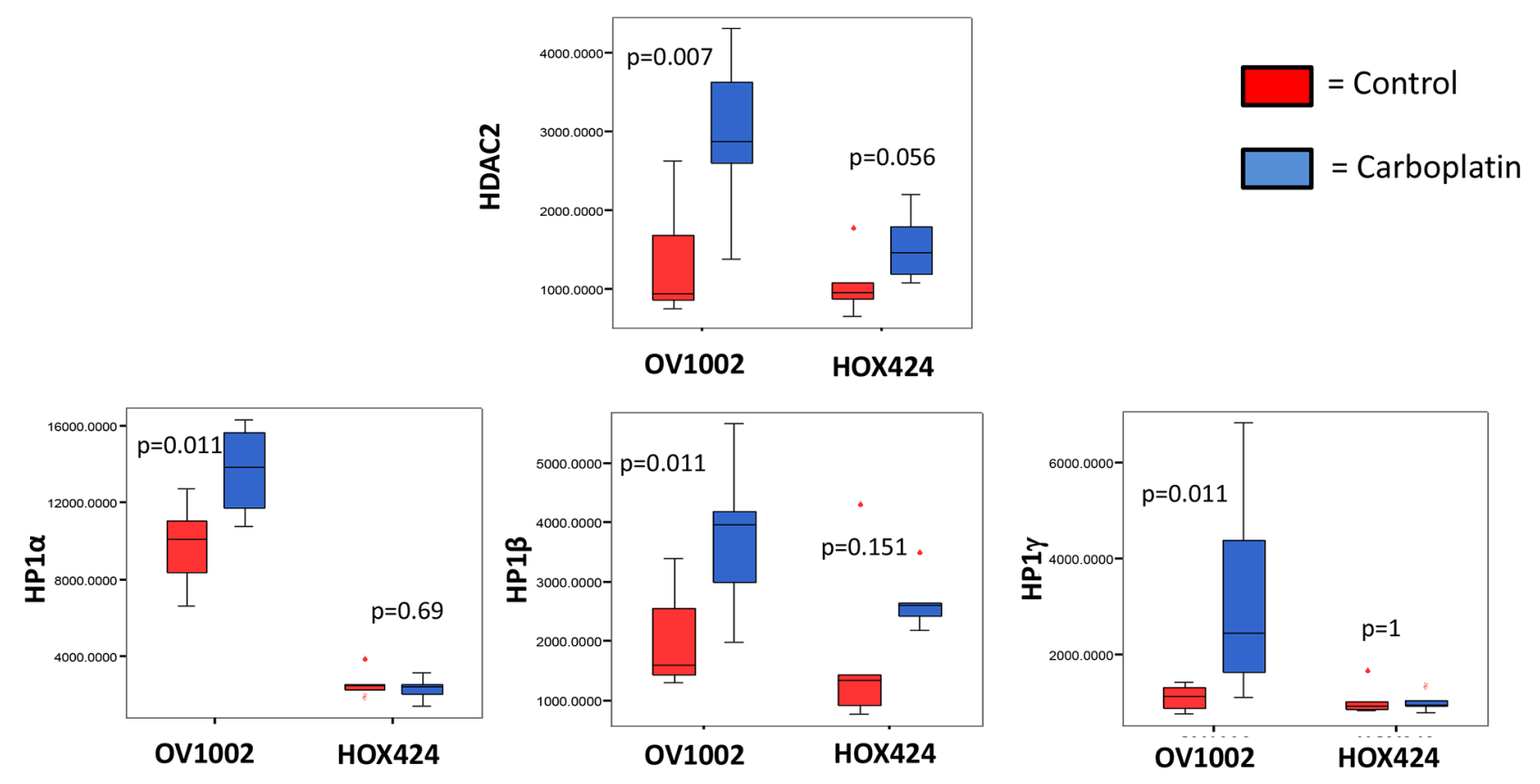

Figure 4. Expression of HDAC2, HP1 $\alpha$, HP1 $\beta$ and HP1 $\gamma$ in the OV1002 and HOX424 ovarian xenograft tumor models on Day 7 after carboplatin treatment. The sample TMA was probed with the indicated antibodies and expression levels were quantified by AQUA analysis. Boxplots depict AQUA scores representing the expression of proteins. Data was compared between control group (red bar) and carboplatin-treated group (blue bar) within the platinum sensitive model (OV1002) and resistant model (HOX424), respectively. Man-Whitney analysis was performed and $\mathrm{P}$ values are indicated. 
the effect of HDAC2 knockdown by siRNA (Figure 6A). HDAC2 knockdown was efficient (Supplementary Figure 1). Expression of HDAC3, HDAC4, and HDAC8 were not significantly affected by HDAC2 knockdown. Interestingly, HDAC1 expression was mildly upregulated after HDAC2 knockdown, indicating a possible compensatory effect as previously reported [35 - 37]. Since HDAC2 was implicated in heterochromatin formation, we further assessed HP isoform expression after HDAC2 knockdown (Figure 6A). There were minor changes in HP1 protein expression, with mild downregulation $(20 \%)$ of HP1 $\alpha$ on HDAC2 knockdown. With respect to DNA damage response protein expression, HDAC2 knockdown resulted in marked upregulation of $\gamma \mathrm{H} 2 \mathrm{AX}$ and downregulation of pBRCA1 (Figure 6A). In contrast, other DDR proteins (pATM, ATM, pATR, ATR, and Rad51; Figure 6A) were not obviously affected. This suggests that double-strand breaks accumulate and DNA repair might be suppressed on HDAC2 knockdown, although the upregulation of $\gamma \mathrm{H} 2 \mathrm{AX}$ might also indicate that the cells are undergoing apoptosis [38].

\section{Characterisation of cellular responses to cisplatin treatment in ovarian cancer cells when HDAC2 is suppressed}

We next investigated the potential role of HDAC2 in cisplatin response. PE01 cells were treated with cisplatin after HDAC2 knockdown (Figure 6B). HDAC2 was consistently upregulated at $24 \mathrm{~h}$ and downregulated at later time points in response to cisplatin without HDAC2 knockdown. Unsurprisingly, the induction of doublestrand breaks (DSBs) indicated by $\gamma \mathrm{H} 2 \mathrm{AX}$ expression occurred as early as $6 \mathrm{~h}$, while $\gamma \mathrm{H} 2 \mathrm{AX}$ expression increased after $24 \mathrm{~h}$ of cisplatin treatment in cells depleted of HDAC2 compared to cisplatin-treated or HDAC2 knockdown groups. This accumulation diminished over 72 h. As expected, pBRCA1, pATM, pATR, and Rad51 participated in the DNA damage response triggered by cisplatin at certain time points $(6 \mathrm{~h}, 24 \mathrm{~h}, 48 \mathrm{~h}$, and $24 \mathrm{~h})$ and were upregulated. In contrast, cisplatin reduced expression of these proteins in cells with HDAC2 knockdown from $24 \mathrm{~h}$ to $72 \mathrm{~h}$ (Figure $6 \mathrm{~B}$ ).

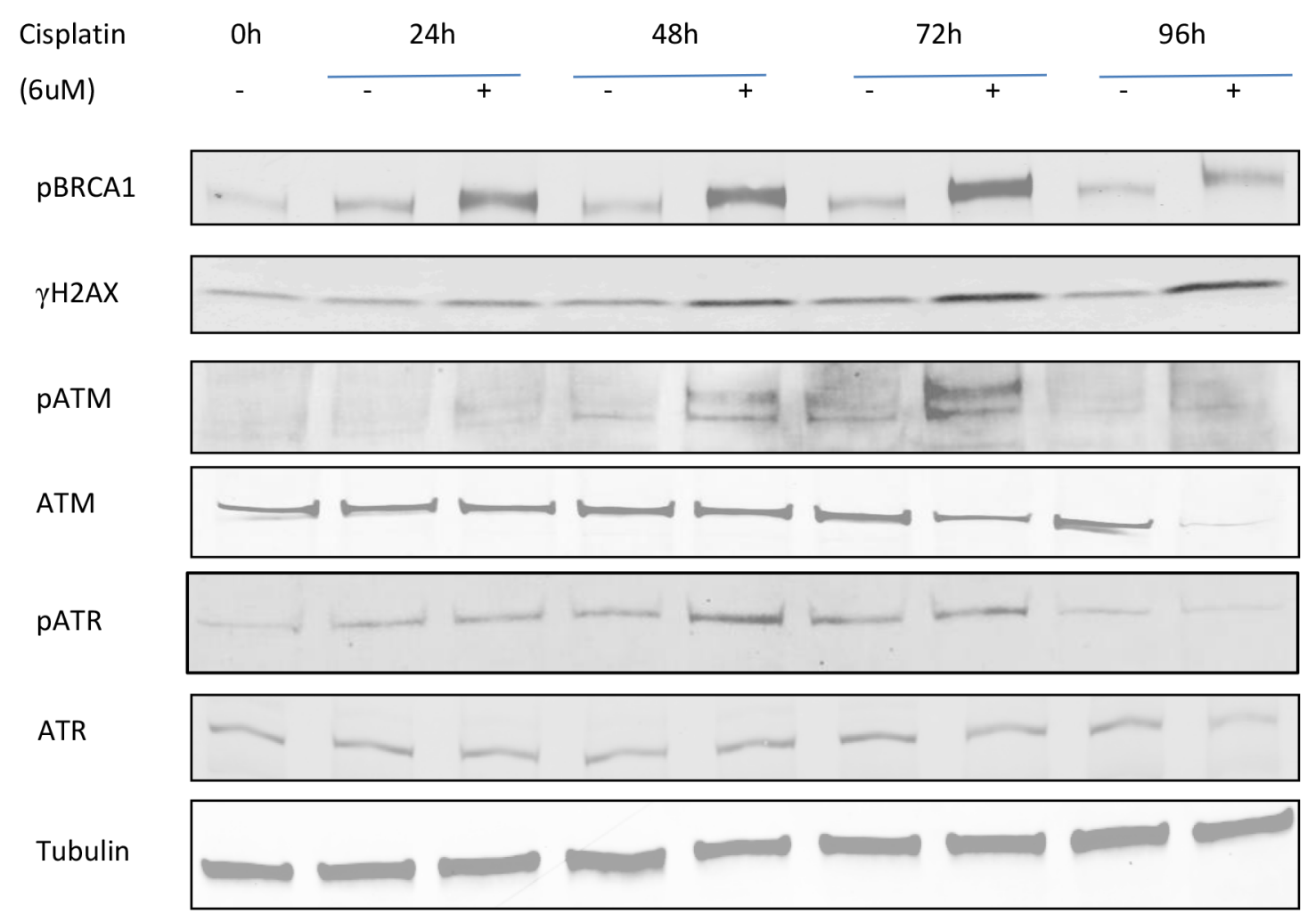

Figure 5. Time-dependent expression of DNA damage response proteins in PEO1 cells. Cells were seeded and treated with or without cisplatin $(6 \mu \mathrm{M})$, and protein lysates were collected every $24 \mathrm{~h}$ after treatment from $0 \mathrm{~h}$ to $96 \mathrm{~h}$. Western blotting was performed to detect expression of pBRCA1, $\gamma \mathrm{H} 2 \mathrm{AX}$, pATM, ATM, pATR, and ATR. Membranes were probed with the indicated antibodies, and tubulin was used as a loading control. Experiments were performed at least three times acquiring similar results. Blots from one representative experiment are shown. 
The cisplatin-resistant PEO4 cell line was next studied to further clarify the involvement of HDAC2 during cisplatin-induced DNA damage responses (Supplementary Figure 2). There was little change in HDAC2 expression in PEO4 cells during cisplatin treatment, except for decreased expression at $72 \mathrm{~h}$. In contrast to PEO1 cells, the cumulative effect of HDAC2 depletion and cisplatin treatment on $\gamma \mathrm{H} 2 \mathrm{AX}$ was not observed in PEO4 cells, although induction of DSBs was noted from $24 \mathrm{~h}$ in response to cisplatin alone. Additionally, pBRCA1, pATM, and RAD51 were upregulated by cisplatin treatment alone. Expression of these proteins was suppressed by HDAC2 depletion, although the pattern of expression over time was different to PEO1 cells.

\section{Role of HDAC2 in $\gamma \mathrm{H} 2 \mathrm{AX}$ foci formation during cisplatin treatment}

$\gamma \mathrm{H} 2 \mathrm{AX}$ foci at DNA damaged domains are indicative of inter-strand crosslinking by cisplatin [39]. The detection of ser139-phosphorylated $\gamma \mathrm{H} 2 \mathrm{AX}$ foci has been widely used to evaluate double-strand breaks (DSBs) [40]. Therefore, $\gamma \mathrm{H} 2 \mathrm{AX}$ foci were targeted and visualised using immunofluorescence microscopy (Figure 7). $\gamma \mathrm{H} 2 \mathrm{AX}$ foci were formed in PEO1 (Figure 7) and PE04 cells in response to cisplatin (Supplementary Figure 3). Similarly, HDAC2 knockdown resulted in $\gamma \mathrm{H} 2 \mathrm{AX}$ foci in both cell lines (Figure 7 and Supplementary Figure 3). There was a marked increase (number and intensity) in $\gamma \mathrm{H} 2 \mathrm{AX}$ foci in PEO1 cells treated with cisplatin after HDAC2

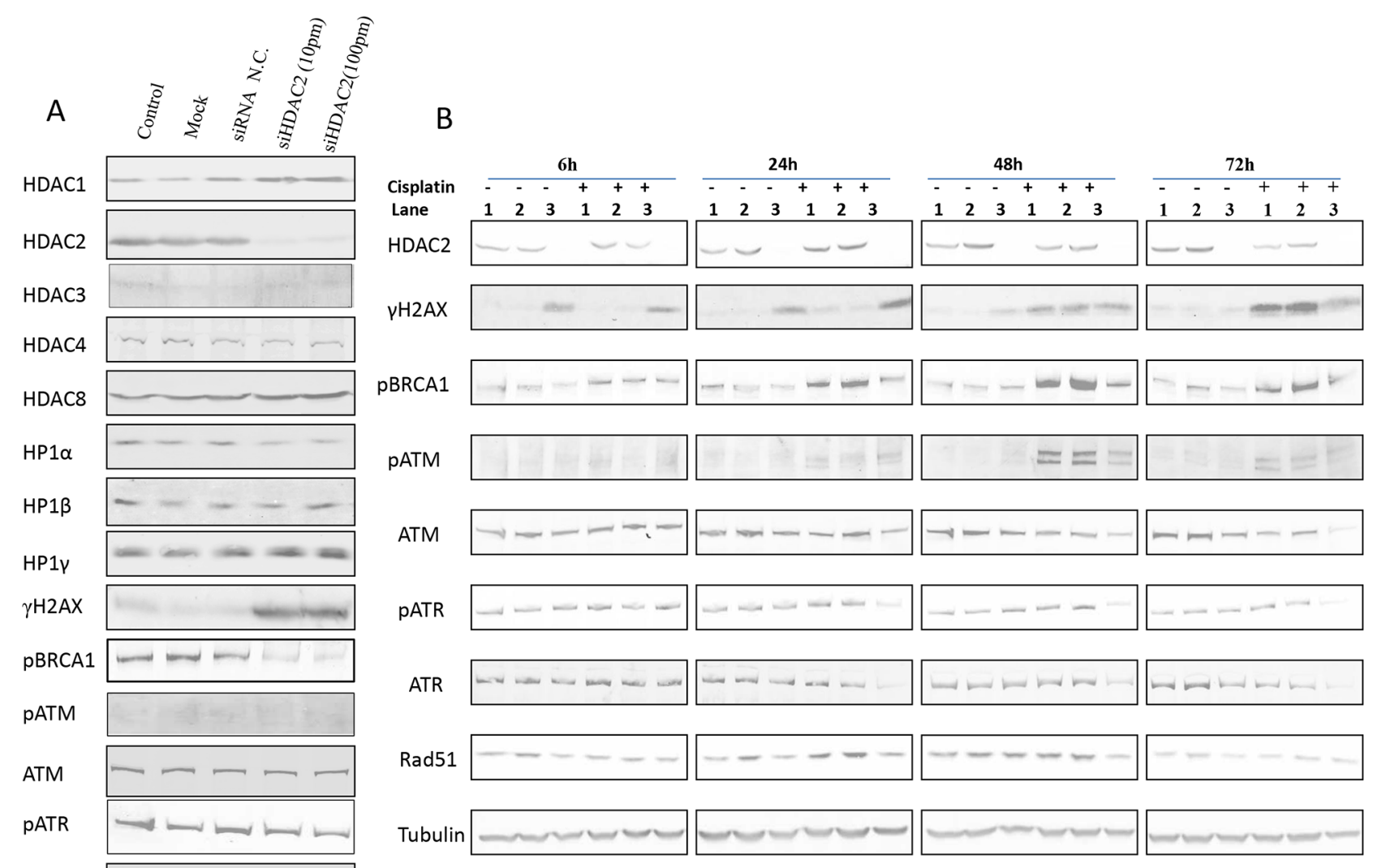

Lane: 1 mock; 2 negative control; 3 siRNA

Figure 6. The effect of siHDAC2 knockdown on expression of other HDAC family members, HP1s, and DNA damage response proteins by western blotting in PEO1 cells alone (A) and in the presence / absence of cisplatin (B). Protein was lysed after HDAC2 was knocked down after $72 \mathrm{~h}$ using reverse transfection. Non-transfection (control), mock, and siRNA negative control were used as controls. Membranes were probed with the indicated antibodies, and tubulin was used as a loading control. Experiments were performed at least three times acquiring similar results. Blots from one representative experiment are shown. In (B), cells were treated with cisplatin $(6 \mu \mathrm{M})$ after $72 \mathrm{~h}$ incubation with mock (lane 1), siRNA negative control (lane 2), or HDAC2 siRNA duplexes (10pmol in 6mL, lane 3). Protein lysates were collected at $6 \mathrm{~h}, 24 \mathrm{~h}, 48 \mathrm{~h}$, and $72 \mathrm{~h}$ after cisplatin treatment and analysed by western blotting of DNA damage response proteins; tubulin was used as loading control. Experiments were performed at least three time acquiring similar results. Blots from one representative experiment are shown. 
knockdown, which was less pronounced in PEO4 cells, similar to cisplatin alone (Supplementary Figure 3). Using a cut-point of five foci per cell as negative background, the number of foci-positive PEO1 cells significantly increased after cisplatin treatment, HDAC2 knockdown, and cisplatin treatment in HDAC2-depleted cells $(\mathrm{p}<0.05$ and 0.01 compared to cisplatin-treated and siRNA-transfected groups, respectively; Figure 7). In PE04 cells, cisplatin induced foci $(\mathrm{p}<0.001)$, while HDAC2 knockdown did not.

\section{Cell fate determination by HDAC2 knockdown during cisplatin treatment}

We assessed the effect of HDAC2 knockdown on cell fate with and without cisplatin treatment. Growth inhibition by cisplatin was concentration dependent manner in both control HDAC2 knockdown groups in PEO1 cells (Figure 8A). Cisplatin treatment alone induced significant S-phase arrest in PEO1 cells $(+160 \%, \mathrm{p}<0.001)$ and decreased the number of G1-phase cells $(-30 \%$; $\mathrm{p}<0.05$ ) (Figure 8B). HDAC2 knockdown alone increased the $\mathrm{S}$ phase population $(+120 \%, \mathrm{p}<0.05)$. As expected, cisplatin treatment in HDAC2-depleted cells altered the cell cycle distribution in PEO1 cells, but in a different way: HDAC2 knockdown caused further S-phase arrest induced by cisplatin in PEO1 cell $(+120 \%, \mathrm{p}<0.05)$ and an additional reduction in G1 phase cells $(-20 \%, \mathrm{p}<0.001)$. In contrast, HDAC2 depletion seemed to reduce S-phase accumulation $(-20 \%, \mathrm{p}<0.001)$ and increase the G1-phase population $(+140 \%, \mathrm{p}<0.05)$ based on measurements of cisplatin treated cells without transfection.

We next analysed the effect of cisplatin and HDAC2 knockdown on apoptosis (Figure 8C). Cisplatin induced early apoptosis (annexin V positive only) $(\mathrm{p}<0.001)$. HDAC2 knockdown caused both early and late apoptosis $(\mathrm{p}<0.001)$. Furthermore, HDAC2 depletion induced significant (especially early) apoptosis after cisplatin treatment $(\mathrm{p}<0.001)$.

\section{Reversibility of HDAC inhibition on nuclear morphological changes during cisplatin treatment}

To test if the nuclear structural changes were mediated by HDACs, then specifically HDAC2, we investigated whether the nuclear changes were reversible by applying either the broad-spectrum HDAC inhibitor trichostatin A (TSA) or HDAC2 siRNA in PEO1 cells. When PEO1 cells were treated with TSA, all five texture parameters were altered: angular second moment, correlation, and inverse difference moment

\section{A}
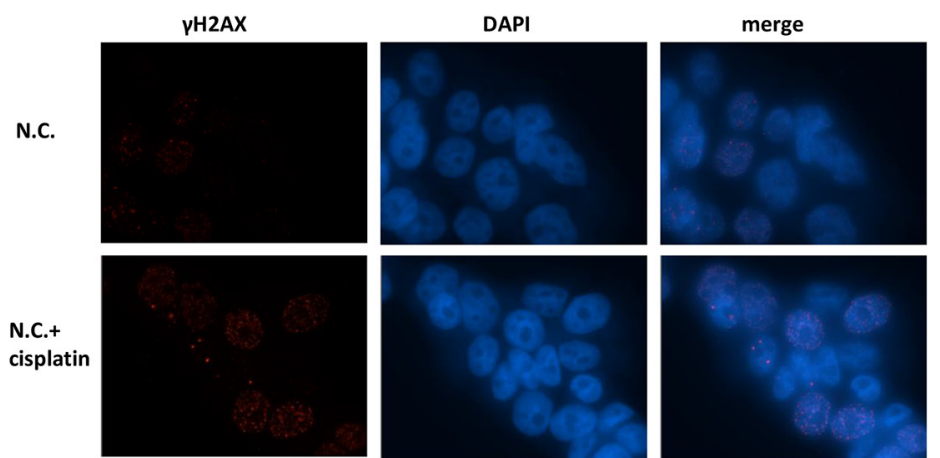

SiRNA
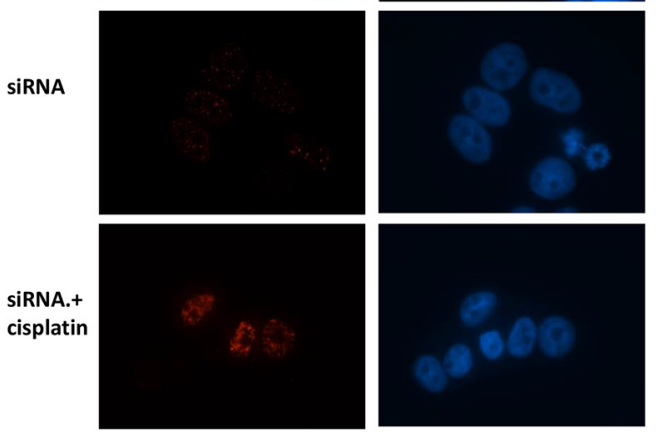
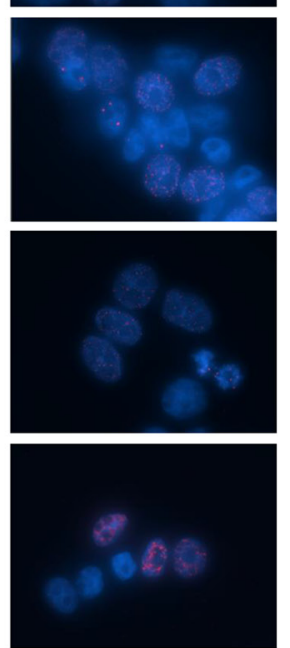

B

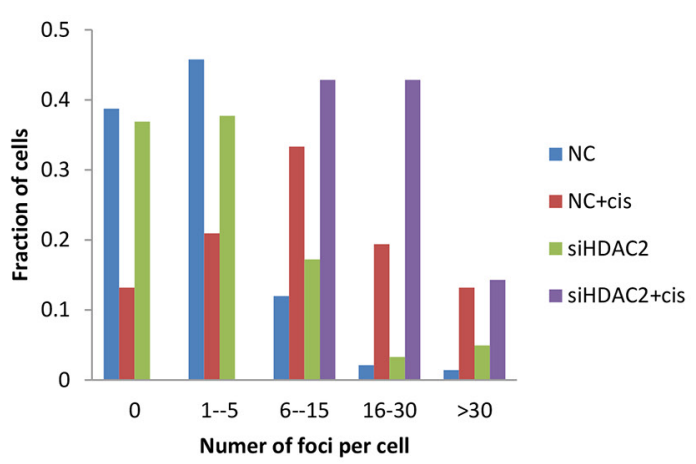

Figure 7. Immunofluorescence for $\gamma \mathrm{H2AX}$ foci in PEO1 cells. Cells were grown and treated as described before, and images were taken after $24 \mathrm{~h}$ treatment of cisplatin in cells with or without HDAC2 siRNA. Antibodies against H2AX phosphorylation at Ser 139 were used to probe cellular $\gamma \mathrm{H} 2 \mathrm{AX}$ foci (red channel), and DAPI was applied for nuclear staining (blue channel). A. Representative images from one experiment are shown. $>100$ cells in each group were included for one experiment, and three independent experiments were performed. B. Distribution of $\gamma \mathrm{H} 2 \mathrm{AX}$ foci / cell in PEO1 cells. 
increased, while entropy and contrast decreased (Figure 9A). Decreased chromatin heterogeneity and contrast and increased homogeneity are consistent with HDAC inhibition relaxing the chromatin structure. With HDAC2 siRNA (Figure 9B), comparable changes to TSA were observed.

\section{DISCUSSION}

Within this study, we show that comparable nuclear morphological changes occur in several ovarian tumor models (cell lines and xenografts) after treatment-induced cellular damage. Image analysis of nuclear texture has previously been used to differentiate between benign and malignant cancers [28], examine apoptotic cells [41], and study the condensation and distribution of chromatin in the nucleus in drug-sensitive and resistant cells [42]. In the present study, increased nuclear heterogeneity was assumed to indicate a transition from euchromatin to heterochromatin. Consistent with this, HP1 isoform expression increased after cisplatin treatment in vitro, which was accompanied by enhanced expression of HDAC2 at both the mRNA and protein levels. Studies in vivo using a patient-derived ovarian cancer xenograft model also showed elevated HDAC2 and HP1 isoform expression after chemotherapy.

As an essential component of heterochromatin, HP1 accumulates in response to UV or ionising radiation consistent with chromatin reorganisation [43]. We obtained a similar result, with enhanced heterochromatin formation following chemotherapy both in vivo and in vitro. HDAC2 was upregulated after $24 \mathrm{~h}$ of cisplatin treatment in vitro, with similar results seen in vivo in ovarian cancer xenografts. In line with its function as a regulator of condensed chromatin formation, HDAC2 expression changed consistent with the observed changes in nuclear texture and was a possible mediator of the DNA damage response. This alteration in chromatin pattern might also indicate that chemotherapy induces transcriptional silencing, perhaps as a form of cellular self-protection upon injury. There is evidence that HDAC-containing complexes including Mi-2/NuRD and/or Sin3/HDAC chromatin-modifying complex (containing HDAC1 and HDAC2) participate in nuclear reorganisation and gene repression during development [44]. Mi-2/NuRD and mSin3/HDAC co-repressor complexes are also necessary for pericentric heterochromatin assembly and chromosome segregation $[45,46]$.

There were time-dependent changes in the expression of DNA damage response proteins including pBRCA1, $\gamma \mathrm{H} 2 \mathrm{AX}, \mathrm{pATM}$, and pATR after cisplatin treatment in PEO1 cells. As a marker of double strand breaks, elevated $\gamma \mathrm{H} 2 \mathrm{AX}$ expression indicated activation of DSB repair pathways in response to cisplatin treatment in PEO1 cells; there is evidence to show that the tumor suppressor function of BRCA1 occurs via heterochromatin silencing, with increased levels of both heterochromatin and BRCA1 observed after DNA damage [47]. Our results are consistent with these findings, and suggest that chromatin remodelling by HDACs is involved in the
A

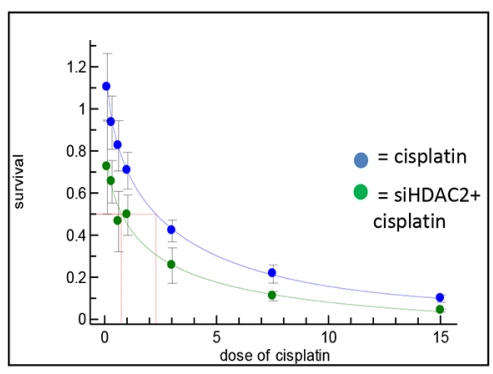

B

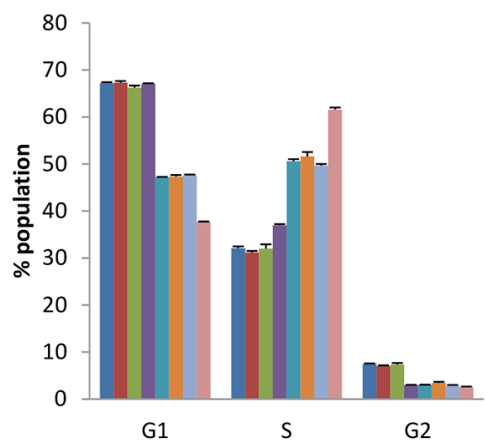

C

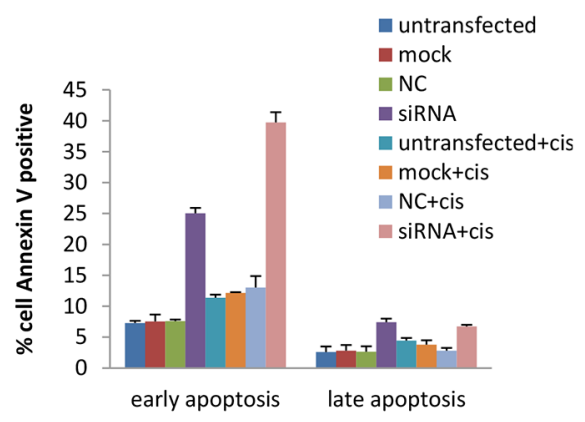

Figure 8. The effect of HDAC2 knockdown on cell number (A), cell cycle distribution (B), and apoptosis (C) in PEO1 cells. A SRB assay profile for growth inhibition of cisplatin on PEO1 cells with (green line) and without (blue line) HDAC2 knockdown. Cells were reverse transfected with HDAC2 siRNA, followed by treatment with cisplatin for $72 \mathrm{~h}$. Three controls were included as described before. B. Percentages of the populations selected in G0/1, S, G2/M phases of the cell cycle were detected by flow cytometry. Columns represent the mean percentage of triplicate independent samples. Error bars represent SD. The Brown-Forsythe test followed by GamesHowell post-hoc test were performed to compare groups for each phase. C. The effect of HDAC2 knockdown on apoptosis in PEO1 cells using the annexin $\mathrm{V}$ assay is shown. Cell number percentages of the population selected with positive annexin $\mathrm{V}$ staining were detected by flow cytometry after cisplatin treatment for $72 \mathrm{~h}$, and data are separated into early and late apoptosis based on propidium Iodide (PI) signal. Columns represent the mean percentage of triplicate independent samples. Error bars represent SD. One-way ANOVA analysis was performed to compare data among groups, and the Tukey HSD pot HOC test was used to compare groups. 
cellular response to DNA damage therapy such as double strand break and DNA repair.

HDACs are associated with malignancy and poor clinical outcomes in multiple cancer types [13, 17]. Roles for HDAC2 have been identified in a variety of malignancies. In colon cancer cells, HDAC2 expression has been associated with chemoresistance to genotoxic stress [48]. In neuroblastoma, HDAC2 has been show to act with N-MYC to reduce TP53INP1 expression which influences p53 phosphorylation at serine 46, with subsequent effects on cell proliferation and survival [49].
In leukemia, HDAC2 silencing induces modulation of gene expression leading to strong transcriptional activation [50] while in lung cancer, HDAC2 has been proposed to exert an effect on survival by sustaining Mdm2-survivin levels [51]. A number of studies have demonstrated efficacy of combined HDAC inhibitor and cytotoxic chemotherapy. The HDAC inhibitor romidepsin (FK228), approved for phase I and II trials, enhances the cytotoxic effects of cisplatin by reducing cell growth and inducing more DNA damage-induced cell death in vitro and in vivo [52]. Since HDAC2 in particular was associated with

A.

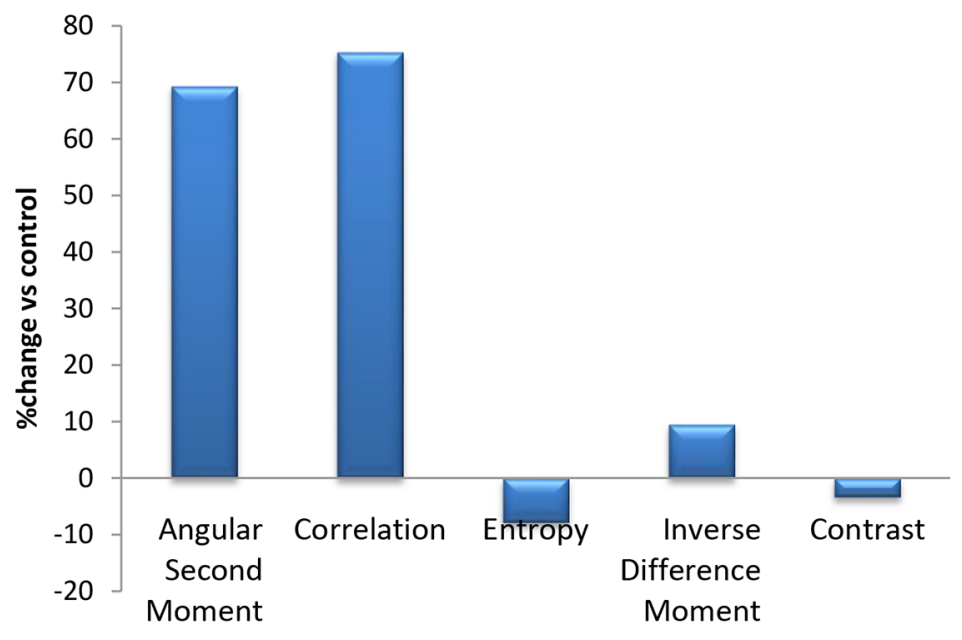

B.
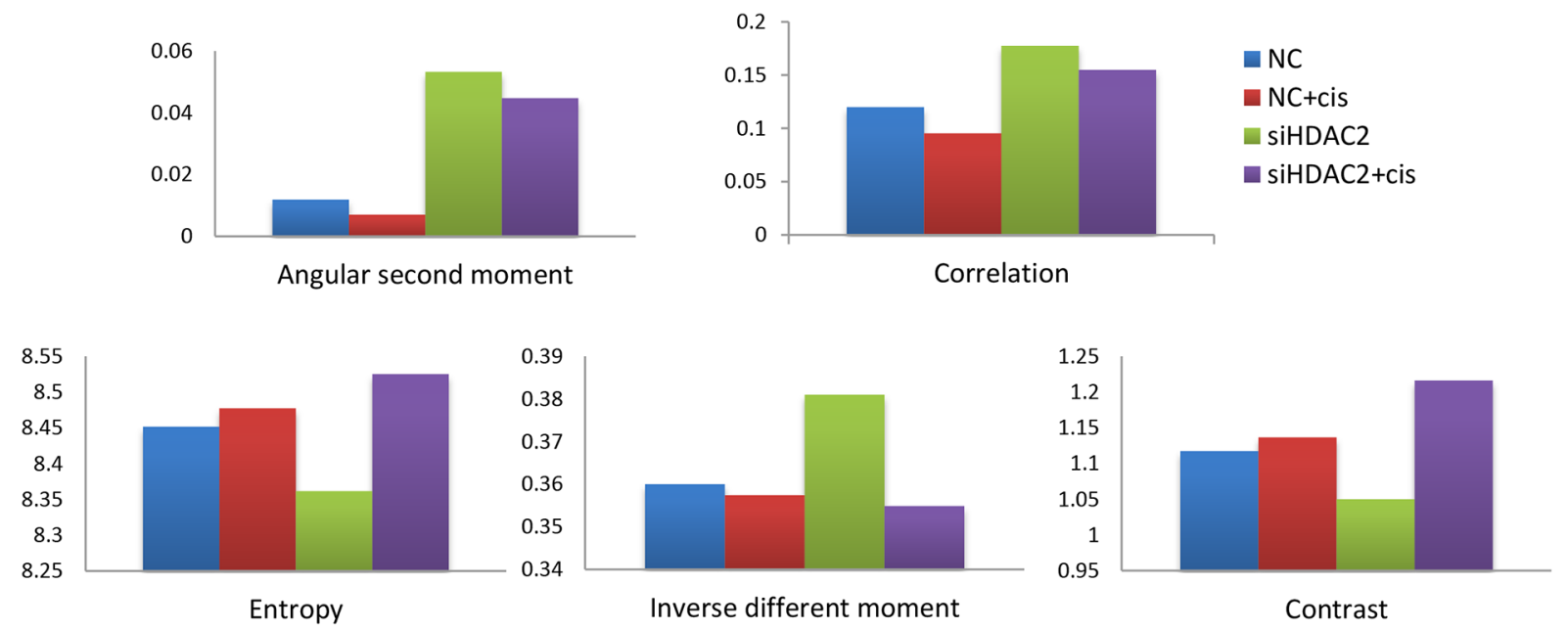

Figure 9. Changes in nuclear texture features in PEO1 cells treated with TSA (A) or transfected with HDAC2 siRNA before cisplatin treatment (B). A. PEO1 cells were grown on coverslips and treated with TSA (250nM) for 24h, and nuclei were stained with DAPI for visualisation using a fluorescence microscope. At least 100 nuclei were included in one experiment. Nuclear texture was analysed by measuring five texture parameters (angular second moment, correlation, entropy, inverse different moment, and contrast) in Image J software. Data are presented as the average change (\%) in TSA treated group for each parameter over control group. B. PEO1 cells were grown on coverslips, transfected with HDAC2 siRNA for $72 \mathrm{~h}$, and treated with cisplatin $(6 \mu \mathrm{M})$ for a further $24 \mathrm{~h}$. Nuclei were stained with DAPI for visualisation using fluorescence microscopy. At least 100 nuclei were included in each group in one experiment. Nuclear texture was analysed by measuring five texture parameters (angular second moment, correlation, entropy, inverse different moment, and contrast) using Image J. 
early responses to cisplatin in our cell culture studies, cell growth was evaluated in response to HDAC2 knockdown. HDAC2 depletion reduced the $\mathrm{IC}_{50}$ of cisplatin in PEO1 cells, suggesting that HDAC2 loss enhances the effect of cisplatin treatment. Repression of cell growth in chemosensitive PEO1 cells by cisplatin after HDAC2 silencing appeared to be due to accumulated S-phase arrest and apoptosis.

Cell cycle progression blockade has been reported to occur as a result of checkpoint activation during DNA damage-based therapy, and at least one checkpoint protein, Chk1, is upregulated during intra-S-phase accumulation by affecting chromatin formation and interfering with the initiation and elongation of DNA replication [53]. It is feasible that HDAC2 interacts with checkpoint proteins to prevent DNA replication by condensing the chromatin in self-protection, and triggers apoptosis via distinct pathways in platinum sensitive and resistant cells. Several mechanisms have been suggested to explain HDAC inhibitor-induced apoptosis, such as altered transcription and DDR and DNA damage repair [54-56]. The early upregulation (after $24 \mathrm{~h}$ ) of HDAC2 in sensitive cells may suggest that HDAC2 is acting as a sensor of DNA damage and a trigger of downstream DDR events (such as activation of ATM, ATR, and BRCA1) and chromatin remodelling, followed by histone hyperacetylation to relax the chromatin structure and facilitate recruitment of more DDR mediators to the damaged site.

DSBs (measured by $\gamma \mathrm{H} 2 \mathrm{AX}$ ) accumulated after $24 \mathrm{~h}$ of cisplatin treatment in HDAC2-depleted PEO1 cells, but not PEO4 cells. High $\gamma \mathrm{H} 2 \mathrm{AX}$ expression has previously been associated with cell viability and apoptosis in ovarian cancer [57], which was attributed to the cell type tested and time after damage. $\mathrm{H} 2 \mathrm{AX}$ activation has also been noted a number of days after treatment with an HDAC inhibitor, suggesting that increased $\gamma \mathrm{H} 2 \mathrm{AX}$ expression precedes cancer cell death [58]. Suppression of DDR activation (as evidenced by pATM, pATR, pBRCA1, and RAD51 expression) after HDAC2 knockdown strongly suggests that HDAC2 is involved in responses to DNA damage-based treatment. The differences between PEO1 and PEO4 cells in DSB accumulation and time of pathway activation or suppression is consistent with the observed differences in cell cycle progression, and also might be due their differences in cisplatin sensitivity. The two major pathways of DSB repair, non-homologous end joining (NHEJ) and homologous recombination (HR), appear to compensate for each other [59], and their balance might be disrupted by the known BRCA2 deficiency in PEO1 and secondary mutation to restore BRCA2 in PE04 [60]. This might influence the dominant mechanism of repair in the two cell lines and cause differences in the observed functional activity of the measured DNA damage response proteins after cisplatin treatment.

Together, these results suggest that chromatin remodelling caused by increased HDAC2 expression might be an early cellular event (within $24 \mathrm{~h}$ ) in response to DNA damage. We postulate that, in sensitive tumors, early alterations in chromatin induced by chemotherapy and mediated by histone deacetylation are a form of cellular self-defence to injury by repressing transcription, initiating chemotherapy-triggered DDR, and promoting survival. This is followed by a change to a relaxed chromatin conformation by histone hyperacetylation, such as via $\mathrm{H} 3 \mathrm{k} 56 \mathrm{Ac}$ and $\mathrm{H} 4 \mathrm{k} 16 \mathrm{Ac}$, to provide accessibility of DNA to downstream proteins at damaged sites [61 63]. However, resistant tumors behave differently in terms of their response to DNA damage to chemotherapy; this might be due to their initial chromatin environment or due to other changes in the components of the DDR response pathways in which HDACs participate. In one study, HDAC1/2-associated immediate histone hypoacetylation occurred after laser microirradiation of a human osteosarcoma cell line to promote NHEJ, which was followed by hypoacetylation to enhance HR and guard genome integrity [64], supporting our hypothesis. Linkage between HDAC2 modulation and $\mathrm{H} 4 \mathrm{k} 16$ acetylation has been shown in breast cancer [65].

By identifying the detailed roles that HDACs play in DNA damage responses by remodelling chromatin, we hope to better understand the molecular processes that underpin nuclear structure and identify novel mechanisms that control responses to chemotherapy.

\section{MATERIALS AND METHODS}

\section{Cell culture}

Cisplatin-sensitive PE01 and cisplatin-resistant PE04 ovarian cancer cell lines derived in our laboratory [30] were cultured as monolayers in RPMI 1640 supplemented with $10 \%$ heat-inactivated foetal calf serum (FCS) and penicillin/streptomycin (100 IU/mL) in 5\% $\mathrm{CO}_{2}$ at $37^{\circ} \mathrm{C}$.

\section{Ovarian cancer xenografts}

Two ovarian cancer patient-derived xenograft models were previously established in our laboratory: OV1002 and HOX424 [66]. Female adult CD-1 nude mice housed in individually ventilated cages were treated with carboplatin $(50 \mathrm{mg} / \mathrm{kg}$ i.p.) on day 0 , and tumor samples were collected on days $0,1,4,7$, and 14 after treatment. Tumors were formalin fixed and paraffin embedded (FFPE). The OV1002 xenograft model was markedly more sensitive to carboplatin treatment than the HOX424 model [31]. Xenograft studies were undertaken under a UK Home Office Project Licence in accordance with the Animals (Scientific Procedures) Act 1986, and the University of Edinburgh Animal Ethics Committee approved the study protocol. 


\section{Nuclear texture analysis}

Confocal microscopy images were obtained using a Nikon A1R Confocal Microscope (Nikon Corporation, Tokyo, Japan) and images were viewed in NIS Viewer (Nikon). Cells were grown on chamber slides for the periods specified and fixed in 10\% formalin in PBS for 10 min. After washing three times with PBS- $0.05 \%$ Tween 20 , slides were counterstained with anti-fade reagent with DAPI (Invitrogen, P36931; Thermo Fisher Scientific, Waltham, MA). Nuclear texture was analysed using Image J software. Briefly, after input of 8-bit images, fully focused areas containing tumor cells were marked as regions of interest (ROI) and individual nuclei selected. Nucleus counter and GLCM (grey-level co-occurrence matrix) [67] manager plugins were performed on each image and five parameters associated with texture obtained: correlation, contrast, angular second moment (ASM), inverse different moment (IDM), and entropy. GLCM $[67,68]$ is a secondorder texture calculation that considers the distance and angle relationship between two-pixel groups in the original greyscale image under the same intensity of grey pixels within a defined area. Texture features were regarded as positively or negatively associated with chromatin patterns (homogeneity, heterogeneity, and contrast) as previously described [28, 29].

\section{siRNA knockdown of HDAC2}

Loss of HDAC2 function was achieved with siRNA transfection of cells in $60 \mathrm{~mm}$ cell culture dishes according to the manufacturer's instructions. Briefly, siRNA duplex-Lipofectamine ${ }^{\mathrm{TM}}$ RNAiMAX complexes (Life Technologies, Thermo Fisher Scientific) were prepared as follows. 10-100 pmol siRNA duplex was diluted in $500 \mu \mathrm{l}$ Opti-MEM $^{\circledR}$ I Medium (Life Technologies) without serum in each cell culture dish and mixed gently. The duplex sequences were: GACAAACCAGAACACUCCAGAAUAU and AUAUUCUGGAGUGUUCUGGUUUGUC. A negative scrambled stealth siRNA duplex with similar GC content (low GC duplex) to the target was used as control. 0.8 $\mu$ lipofectamine ${ }^{\mathrm{TM}}$ RNAiMAX was added to each dish containing diluted siRNAs and incubated at room temperature for $20 \mathrm{~min}$. After incubation, cells were diluted in complete growth medium without antibiotics at $700,000-800,000$ cells $/ 5 \mathrm{~mL}$ to ensure a cell density of $30-50 \% 24 \mathrm{~h}$ after seeding, and $5 \mathrm{~mL}$ was added to each well. Controls were untransfected (no transfection agents), mock (only Lipofectamine ${ }^{\mathrm{TM}}$ RNAiMAX mixture), and negative control (random RNAi duplex). The cells and the complexes were incubated for $24-120 \mathrm{~h}$ at $37^{\circ} \mathrm{C}$ in full serum without antibiotics. For drug treatment, cisplatin was added after $48 \mathrm{~h}$ of transfection and cells were collected after several time points as indicated.

\section{Protein extraction from mammalian cell lines}

Cultured cells were washed in cold PBS and lysed by scraping in ice-cold isotonic lysis buffer $(50 \mathrm{mM}$ Tris$\mathrm{HCl}$ (pH7.5), 5mM EGTA (pH 8.5), $150 \mathrm{mM} \mathrm{NaCl}, 1 \%$ Triton X-100) supplemented with aprotinin $(10 \mu \mathrm{g} / \mathrm{mL})$ and a cOmplete ${ }^{\mathrm{TM}}$ Protease Inhibitor Cocktail Tablet (Roche, 11836153001) for $30 \mathrm{~min}$ on ice. Lysates were centrifuged for $6 \mathrm{~min}$ at $13,000 \times \mathrm{g}$ and the supernatant stored at $-70^{\circ} \mathrm{C}$. Protein concentrations were determined using the bicinchoninic acid (BCA) assay (Sigma, BCA-1; Sigma-Aldrich, St. Louis, MO).

\section{Western blotting}

After SDS-PAGE using 10\% polyacrylamide gels, resolved proteins were transferred to nitrocellulose membranes at $30 \mathrm{~V}, 4^{\circ} \mathrm{C}$ overnight. After transfer, membranes were rinsed in PBST and blocked with $\mathrm{Li}$ Cor Odyssey Blocking Buffer (LI-COR Biosciences, Lincoln, NE; diluted 50:50 in PBS) for $1 \mathrm{~h}$ at room temperature before probing overnight at $4^{\circ} \mathrm{C}$ with the appropriate primary antibody in Li-Cor Odyssey Blocking Buffer. Primary antibodies were rabbit and obtained from Cell Signaling Technology (Beverly, MA) and used at 1:1000 unless otherwise indicated: anti-HP1 alpha (\#2623), anti-HP1 beta (Abcam/ab10478), antiHP1 gamma (\#2619), anti-HDAC1 (mouse, \#5356), anti-HDAC2 (\#2540), anti-HDAC3 (Abcam (Cambridge, UK) ab32369), anti-HDAC4 (Abcam ab32534), antiHDAC8 (Abcam ab39664), anti-AMT (1:750 Abcam ab67998, mouse), anti-pATM (Ser1981; \#4526), antiATR (Abcam ab2905), anti-pATR (Ser428; \#2853), anti-pBRCA1(Ser1524; \#9009), anti- $\gamma H 2 A X ~(\# 2577)$, anti-Rad51 (H-92) (Santa Cruz Biotechnology, Dallas, TX; sc-8349), anti- $\alpha$-tubulin (1:6000 Mouse Abcam ab7291), anti- $\beta$-tubulin (1:6000 Abcam ab6046), antiGAPDH (1:8000 Mouse Abcam ab8245). Membranes were washed with PBS-Tween 20 before incubation with fluorescently-labelled secondary antibodies diluted in Odyssey Blocking Buffer (50:50 in PBST) at 1:10,000 dilution. Mouse-derived primary antibodies were detected using an anti-mouse fluorescently-labelled secondary antibody (680 nm wavelength), whilst rabbitderived primary antibodies were detected using an antirabbit fluorescently-labelled secondary antibody $(800 \mathrm{~nm}$ wavelength) (45 min incubation). By combining a mouse primary with a rabbit primary along with their respective secondary antibodies, dual-labelled blots were obtained. Membranes were scanned on the Li-Cor Odyssey scanner, and the fluorescence value (integrated intensity, I.I.) corresponded to protein expression levels. Alphatubulin (Mouse Abcam ab7291) was used as loading control. 


\section{RNA preparation and real time polymerase chain reaction (RT-PCR)}

Total RNA was extracted from cultured cells using the Qiagen Mini RNeasy Kit according to the manufacturer's instructions (Qiagen, Limburg, NL). The concentration and quality of RNA were assessed by NanoDrop. $1 \mu \mathrm{g}$ of total RNA from each individual sample was reverse transcribed using the QuantiTect Reverse Transcription kit (Qiagen) following the manufacturer's instructions to produce $20 \mu \mathrm{L}$ of cDNA, which was quantified using Rotorgene (Corbett Research, San Francisco, CA) and the QuantiTect SYBR Green system (Qiagen) following the manufacturers' instructions. For PCR, a 13-fold dilution of the cDNA mixture (10-fold dilution for standard curve) and a 10-fold dilution of primers for HDAC8 and $\beta$-actin (Qiagen) were used. A $15 \mathrm{ul}$ mixture of $7.5 \mu \mathrm{l} 2 \mathrm{xQuantiTect} \mathrm{SYBR}$ Green iMaster Mix, $1.5 \mu \mathrm{L}$ primer mix $(0.3 \mu \mathrm{M}), 2.5 \mathrm{mM}$ of $\mathrm{MgCl}_{2}$, and $1.5 \mu \mathrm{L}$ cDNA was prepared in RNase-free water for the PCR reactions. PCR was performed as follows: $95^{\circ} \mathrm{C}$ for $15 \mathrm{~min} ; 45$ cycles at $94^{\circ} \mathrm{C}$ for $15 \mathrm{~s}, 56^{\circ} \mathrm{C}$ for $30 \mathrm{~s}, 72^{\circ} \mathrm{C}$ for $30 \mathrm{~s} ; 72^{\circ} \mathrm{C}$ for $5 \mathrm{~min}$ followed by melting from $55^{\circ} \mathrm{C}$ to $95^{\circ} \mathrm{C}$ at $0.2^{\circ} \mathrm{C} / \mathrm{s}$.

\section{Immunofluorescence (IF) on xenografts}

$4 \mu \mathrm{m}$ TMA sections were deparaffinised in xylene for $5 \mathrm{~min}$ and rehydrated through graded ethanol. For antigen retrieval, sections were treated with $0.15 \mathrm{mM}$ sodium citrate, $\mathrm{pH} 6.0$ or Tris-EDTA, pH9.0 using a microwave pressure cooker for $5 \mathrm{~min}$. Sections were rinsed in $0.05 \%$ PBST, blocked in 3\% hydrogen peroxide and serumfree protein block (Dako, Glostrup, Denmark; \#X0909), 10 min each. After blocking, slides were incubated with primary antibodies diluted in second primary antibody (mouse anti-cytokeratin, Invitrogen, \#18-0132) in Dako antibody diluents $(1: 25)$ for $1 \mathrm{~h}$ at room temperature or overnight at $4{ }^{\circ} \mathrm{C}$. Primary antibodies were rabbit from Cell Signaling unless otherwise indicated: anti-HP1 alpha (\#2623; 1:100 dilution), anti-HP1 beta (Abcam ab10478; 1:150), anti-HP1 gamma (\#2619; 1:400 dilution), antiHDAC1 (mouse \#5356; 1:25 dilution), anti-HDAC2 (\#2540; 1/100 dilution), anti-HDAC3 (Abcam ab32369; 1/100 dilution), anti-HDAC4 (Abcam/ab32534; 1/100 dilution) and anti-HDAC8 (Abcam/ab39664; 1/100 dilution). Sections were rinsed in $0.05 \%$ PBST three times followed by incubation with secondary antibodies for $1 \mathrm{~h}$ at room temperature with a 1:25 dilution of goatanti-mouse Alexa 555 antibody (Invitrogen, \#A21422). After rinsing, sections were incubated with target signal amplification diluents and Cy5 tyramide at 1:50 in the dark for $10 \mathrm{~min}$ at room temperature for target visualisation. Finally, slides were rinsed, dehydrated in $80 \%$ ethanol for $1 \mathrm{~min}$, air dried in the dark, and counterstained and coverslipped using Prolong Gold anti-fade reagent with DAPI (Invitrogen, P36931).
IF was analysed using the Automated QUantitative Analysis (AQUA) system (HistoRx, New Haven, CT) as previously described [69]. For each immunofluorescence image, AQUAnalysis software evaluated the quantity (in AQUA units $=\mathrm{Au}$ ) of target protein expression (through Cy-5-tyramide) within the cytoplasm (identified by cytokeratin) and nuclei (DAPI). Images were examined to exclude imaging faults and normal tissue, thus target protein expression was scored only in invasive cancers. Cores containing $<5 \%$ epithelium were automatically excluded to ensure tissues were representative of tumors [70]. The final normalised AQUA score detecting the fluorescence correlates with the expression level of target protein.

\section{Sulforhodamine B cell proliferation assay}

Cells were harvested in log phase, counted using a haemocytometer, and optimal initial numbers of cells in $200 \mathrm{uL}$ per well were seeded into 96 well cell culture plates for $72 \mathrm{~h}$. After 0 to 6 days incubation with small molecules, cells were fixed in $25 \%$ cold trichloroacetic acid $(50 \mathrm{uL} / \mathrm{well})$, and incubated for $1 \mathrm{~h}$ at $4^{\circ} \mathrm{C}$. Plates were washed, air-dried, and stained with sulforhodamine B dye $(0.4 \%$ solution in $1 \%$ acetic acid, $50 \mu \mathrm{l} /$ well) for 30 min. After washing with $1 \%$ acetic acid, plates were dried. $100 \mathrm{uL}$ Tris buffer $(10 \mathrm{mM}, \mathrm{pH} 10.5)$ was added into each well $1 \mathrm{~h}$ prior to reading and the optical density (OD) was recorded using a Biohit BP800 Microplate reader (Biohit, Helsinki, Finland) at $540 \mathrm{~nm}$.

\section{Cell cycle analysis}

Cells were harvested and plated as described for western blotting. At the time points indicated, cells were trypsinised and transferred to $5 \mathrm{~mL}$ BD Falcon tubes (BD Biosciences). Citrate buffer (trisodium citrate (301287F, BDH Laboratory Supplies, Poole, UK), $121 \mathrm{mg}$ Tris Base (T1378, Sigma), $1044 \mathrm{mg}$ spermine tetrahydrochloride (S2876, Sigma) and $2 \mathrm{~mL}$ Nonidet NP40 (N3516, Sigma) in 2000mL distilled water, pH7.6) was added after centrifugation. The following solutions were added in sequence prior to analysis: $450 \mathrm{uL}$ solution A $(0.003 \%$ trypsin type IX-S (T0303, Sigma) in citrate buffer, pH7.6) for 2 min, solution B $(0.05 \%$ trypsin inhibitor (T9253, Sigma) and 0.01\% RNAse A (R4875, Sigma) in citrate buffer $\mathrm{pH} 7.6)$ for $10 \mathrm{~min}$, and solution C $(0.0416 \%$ propidium iodide (81845, Sigma) and $0.1 \%$ spermine tetrahydrochloride (S2876, Sigma) in $500 \mathrm{~mL}$ citrate buffer $\mathrm{pH} 7.6$ ) for $10 \mathrm{~min}$ in the dark. Apoptosis was detected at $24 \mathrm{~h}$ using the TACS Annexin V-FITC Kits (R $\& D$ Systems) according to the manufacturer's protocol. Flow cytometry was performed using a BD FACSArialI SORP (Becton Dickinson, Franklin Lakes, NJ). BD FACSDiva software (Becton Dickinson, Version 6.1.2) was used for instrument control and Flowjo software (Version 7.6.5) for Data analysis. 


\section{Quantitative analysis of $\gamma \mathrm{H} 2 \mathrm{AX}$ foci formation in cells}

Cells were grown and stained as described above using anti-phospho-histone H2AX (ser139, $\gamma \mathrm{H} 2 \mathrm{AX}$; Millipore 05-636) primary antibody. After mounting with DAPI and air-drying, cells were visualised with a BriteMAC or MacRd microscope. Nuclear images were taken with IPLab software with single filters for each channel and the same exposure for each set of experiments. For each slide, over 100 nuclei were counted for foci formation. The number of $\gamma \mathrm{H} 2 \mathrm{AX}$ foci in each nucleus was counted by the PZ Foci EZ plugin in ImageJ as described (available at www.pzfociez.com). Briefly, a nuclear mask defining ROIs was first created for each channel, and then the foci number was automatically counted in the channel within the defined ROI.

\section{Statistical analysis}

Student's t-test was used to compare two independent samples. One-way ANOVA followed by the Tukey test was for multiple comparisons of groups with equal variance. Pearson correlations were performed for IF correlations. The Wilcoxon signed-rank test was used to compare target protein expression differences between the pre- and post-treatment samples from patients, and the Mann-Whitney test was performed to compare xenograft data. All data were analysed using GraphPad Prism (GraphPad Software Inc., La Jolla, CA), and a p-value $<0.05$ was considered statistically significant.

\section{ACKNOWLEDGMENTS}

We are grateful to Paul Perry and Matthew Pearson (IGMM, University of Edinburgh) for their help and advice with the confocal microscopy. We are also grateful to Elizabeth Freyer (IGMM, University of Edinburgh) for her help and support of the apoptosis and cell cycle experiments.

\section{FUNDING}

This study was supported by financial support from the University of Edinburgh/China Scholarship Council joint Scholarship Scheme. We are also grateful to support from the Scottish Funding Council.

\section{CONFLICTS OF INTEREST}

The authors declare no conflicts of interest.

\section{REFERENCES}

1. Ho L, Crabtree GR. Chromatin remodelling during development. Nature. 2010; 463: 474-484.
2. Turner B. Histone acetylation and an epigenetic code. Bioessays. 2000; 22: $836-845$.

3. Lafon-Hughes L, Di Tomaso MV, Méndez-Acuña L, Martínez-López W. Chromatin-remodelling mechanisms in cancer. Mutation Res. 2008; 658: 191-214.

4. Kornberg RD, Lorch Y. Twenty-five years of the nucleosome, fundamental particle of the eukaryote chromosome. Cell. 1999; 98: 285-294.

5. Margueron R, Trojer P, Reinberg D. The key to development: interpreting the histone code? Curr Opin Genet Dev. 2005; 15: 163-176.

6. Jeppesen P. Histone acetylation: a possible mechanism for the inheritance of cell memory at mitosis. Bioessays. 1997; 19: 67-74.

7. Hassa PO, Hottiger MO. An epigenetic code for DNA damage repair pathways? Biochem Cell Biol. 2005; 83: 270-285.

8. Williams RR, Azuara V, Perry P, Sauer S, Dvorkina M, Jørgensen H, Roix J, McQueen P, Misteli T, Merkenschlager M, Fisher AG. Neural induction promotes large-scale chromatin reorganisation of the Mash1 locus. J Cell Sci. 2007; 119: 132-140.

9. Gibbons RJ. Histone modifying and chromatin remodelling enzymes in cancer and dysplastic syndromes. Hum Mol Genet. 2005; 14 Spec No 1: R85-92.

10. Fog CK, Jensen KT, Lund AH. Chromatin-modifying proteins in cancer. APMIS. 2007; 115: 1060-1089.

11. Grønbaek K, Hother C, Jones PA. Epigenetic changes in cancer. APMIS. 2007; 115: 1039-1059.

12. de Ruijter AJ, van Gennip AH, Caron HN, Kemp S, van Kuilenburg AB. Histone deacetylases (HDACs): characterization of the classical HDAC family. Biochem J. 2003; 370: 737-749.

13. Acharya MR, Sparreboom A, Venitz J, Figg WD. Rational development of histone deacetylase inhibitors as anticancer agents: a review. Mol Pharmacol. 2005; 68: 917-932.

14. Santos-Rosa H, Caldas C. Chromatin modifier enzymes, the histone code and cancer. Eur J Cancer. 2005; 41: 2381-2402.

15. Vannini A, Volpari C, Filocamo G, Casavola EC, Brunetti M, Renzoni D, Chakravarty P, Paolini C, De Francesco R, Gallinari P, Steinkühler C, Di Marco S. Crystal structure of a eukaryotic zinc dependent histone deacetylase, human HDAC8, complexed with a hydroxamic acid inhibitor. Proc Natl Acad Sci U S A. 2004; 101: 15064-15069.

16. Grønbaek K, Hother C, Jones PA. Epigenetic changes in cancer. APMIS. 2007; 115: 1039-1059.

17. Mahlknecht U, Hoelzer D. Histone acetylation modifiers in the pathogenesis of malignant disease. Mol Med. 2000; 6: 623-644.

18. Johnstone RW, Licht JD. Histone deacetylase inhibitors in cancer therapy: is transcription the primary target? Cancer Cell. 2003; 4: 13-18. 
19. Gui CY, Ngo L, Xu WS, Richon VM, Marks PA. Histone deacetylase (HDAC) inhibitor activation of p21WAF1 involves changes in promoter-associated proteins, including HDAC1. Proc Natl Acad Sci U S A. 2004; 101: 1241-1246.

20. Cheutin T, McNairn AJ, Jenuwein T, Gilbert DM, Singh $\mathrm{PB}$, Misteli T. Maintenance of stable heterochromatin domains by dynamic HP1 binding. Science. 2003; 299: 721-725.

21. Popova EY, Claxton DF, Lukasova E, Bird PI, Grigoryev SA. Epigenetic heterochromatin markers distinguish terminally differentiated leukocytes from incompletely differentiated leukemia cells in human blood. Exp Hematol. 2006; 34: 453-462.

22. Dialynas GK, Vitalini MW, Wallrath LL. Linking Heterochromatin Protein 1 (HP1) to cancer progression. Mutat Res. 2008; 647: 13-20.

23. Minc E, Allory Y, Courvalin JC, Buendia B. Immunolocalization of HP1 proteins in metaphasic mammalian chromosomes. Methods Cell Sci. 2001; 23: 171-174.

24. James TC, Eissenberg JC, Craig C, Dietrich V, Hobson A, Elgin SC. Distribution patterns of HP1, a heterochromatinassociated non histone chromosomal protein of Drosophila. Eur J Cell Biol. 1989; 50: 170-180.

25. Maloney A, Clarke PA, Naaby-Hansen S, Stein R, Koopman JO, Akpan A, Yang A, Zvelebil M, Cramer R, Stimson L, Aherne W, Banerji U, Judson I et al. Gene and protein expression profiling of human ovarian cancer cells treated with the heat shock protein 90 inhibitor 17-allylamino-17-demethoxygeldanamycin. Cancer Res. 2007; 67: 3239-3253.

26. Kirschmann DA, Lininger RA, Gardner LM, Seftor EA, Odero VA, Ainsztein AM, Earnshaw WC, Wallrath LL, Hendrix MJ. Down-regulation of HP1Hsalpha expression is associated with the metastatic phenotype in breast cancer. Cancer Res. 2000; 60: 3359-3363.

27. Ruginis T, Taglia L, Matusiak D, Lee BS, Benya RV. Consequence of gastrin-releasing peptide receptor activation in a human colon cancer cell line: a proteomic approach. J Proteome Res. 2006; 5: 1460-1468.

28. Murata S, Mochizuki K, Nakazawa T, Kondo T, Nakamura N, Yamashita H, Urata Y, Ashihara T, Katoh R. Detection of underlying characteristics of nuclear chromatin patterns of thyroid tumor cells using texture and factor analyses. Cytometry 2002; 49: 91-95.

29. Murata S, Mochizuki K, Nakazawa T, Kondo T, Nakamura N, Yamashita H, Urata Y, Ashihara T, Katoh R et al. Morphological abstraction of thyroid tumor cell nuclei using morphometry with factor analysis. Microsc Res Tech. $2003 ; 61: 457-62$

30. Langdon SP, Lawrie SS, Hay FG, Hawkes MM, McDonald A, Hayward IP, Schol DJ, Hilgers J, Leonard RC, Smyth JF. Characterization and properties of nine human ovarian adenocarcinoma cell lines. Cancer Res. 1988; 48: 6166-6172.
31. Koussounadis A, Langdon SP, Harrison DJ, Smith VA. Chemotherapy-induced dynamic gene expression changes in vivo are prognostic in ovarian cancer. Br J Cancer. 2014; 110:2975-2984.

32. Witt O, Deubzer HE, Milde T, Oehme I. HDAC family: What are the cancer relevant targets? Cancer letters. 2009; 277: 8-21.

33. Hayashi A, Horiuchi A, Kikuchi N, Hayashi T, Fuseya C, Suzuki A, Konishi I, Shiozawa T. Type-specific roles of histone deacetylase (HDAC) overexpression in ovarian carcinoma: HDAC1 enhances cell proliferation and HDAC3 stimulates cell migration with downregulation of E-cadherin. Int J Cancer. 2010; 127: 1332-1346.

34. Stronach EA, Alfraidi A, Rama N, Datler C, Studd JB, Agarwal R, Guney TG, Gourley C, Hennessy BT, Mills GB, Mai A, Brown R, Dina R, Gabra H. HDAC4-regulated STAT1 activation mediates platinum resistance in ovarian cancer. Cancer Res. 2011; 71: 4412-4422.

35. Wilting RH, Yanover E, Heideman MR, Jacobs H, Horner J, van der Torre J, DePinho RA, Dannenberg JH. Overlapping functions of Hdac1 and Hdac2 in cell cycle regulation and haematopoiesis. EMBO J. 2010; 29: 2586-2597.

36. Yamaguchi T, Cubizolles F, Zhang Y, Reichert N, Kohler $\mathrm{H}$, Seiser C, Matthias P. Histone deacetylases 1 and 2 act in concert to promote the G1-to-S progression. Genes Devel. 2010; 24: 455-469.

37. Jurkin J, Zupkovitz G, Lagger S, Grausenburger R, Hagelkruys A, Kenner L, Seiser C. Distinct and redundant functions of histone deacetylases HDAC1 and HDAC2 in proliferation and tumorigenesis. Cell cycle 2011; 10: 406-412.

38. Tanaka T, Kurose A, Huang X, Dai W, Darzynkiewicz Z. ATM activation and histone H2AX phosphorylation as indicators of DNA damage by DNA topoisomerase I inhibitor topotecan and during apoptosis. Cell Proliferation. 2006; 39: 49-60.

39. Clingen PH, Wu JY, Miller J, Mistry N, Chin F, Wynne P, Prise KM, Hartley JA. Histone H2AX phosphorylation as a molecular pharmacological marker for DNA interstrand crosslink cancer chemotherapy. Biochem Pharmacol. 2008; 76: 19-27.

40. Rogakou EP, Pilch DR, Orr AH, Ivanova VS, Bonner WM. DNA double-stranded breaks induce histone H2AX phosphorylation on serine 139. J Biol Chem. 1998; 273: 5858-5868.

41. Pantic I, Pantic S, Basta-Jovanovic G. Gray level co-occurrence matrix texture analysis of germinal center light zone lymphocyte nuclei: physiology viewpoint with focus on apoptosis. Microsc Microanal. 2012; 18:470-475.

42. Yatouji S, El-Khoury V, Trentesaux C, Trussardi-Regnier A, Benabid R, Bontems F, Dufer J. Differential modulation of nuclear texture, histone acetylation, and MDR1 gene 
expression in human drug-sensitive and -resistant OV1 cell lines. Int J Oncol. 2007; 30: 1003-1009.

43. Luijsterburg MS, Dinant C, Lans H, Stap J, Wiernasz E, Lagerwerf S, Warmerdam DO, Lindh M, Brink MC, Dobrucki JW, Aten JA, Fousteri MI, Jansen G et al. Heterochromatin protein 1 is recruited to various types of DNA damage. J Cell Biol. 2009; 185: 577-586.

44. McDonel P, Costello I, Hendrich B. Keeping things quiet: Roles of NuRD and Sin3 co-repressor complexes during mammalian development. Int J Biochem Cell Biol. 2009; 41: $108-116$.

45. David G, Turner GM, Yao Y, Protopopov A, DePinho RA. $\mathrm{mSin} 3$-associated protein, $\mathrm{mSds} 3$, is essential for pericentric heterochromatin formation and chromosome segregation in mammalian cells. Genes Dev. 2003; 17:2396 - 2405.

46. Sims JK, Wade PA. Mi-2/NuRD complex function is required for normal $\mathrm{S}$ phase progression and assembly of pericentric heterochromatin. Mol Biol Cell. 2011; 22: 3094 -3102 .

47. Zhu Q, Pao GM, Huynh AM, Suh H, Tonnu N, Nederlof PM, Gage FH, Verma IM. BRCA1 tumour suppression occurs via heterochromatin mediated silencing. Nature. 2011; 477: 179-184.

48. Wagner T, Kiweler N, Wolff K, Knauer SK, Brandl A, Hemmerich P, Dannenberg JH, Heinzel T, Schneider G, Krämer $\mathrm{OH}$. Sumoylation of HDAC2 promotes NF-кBdependent gene expression. Oncotarget. 2015 ; 6 :7123 -7135. doi: 10.18632/oncotarget.3344.

49. Shahbazi J, Scarlett CJ, Norris MD, Liu B, Haber M, Tee AE, Carrier A, Biankin AV, London WB, Marshall GM, Lock RB, Liu T. Histone deacetylase 2 and N-Myc reduce $\mathrm{p} 53$ protein phosphorylation at serine 46 by repressing gene transcription of tumor protein 53-induced nuclear protein. Oncotarget. 2014; 5: 4257-4268. doi: 10.18632/oncotarget.1991.

50. Conte M, Dell'Aversana C, Benedetti R, Petraglia F, Carissimo A, Petrizzi VB, D’Arco AM, Abbondanza C, Nebbioso A, Altucci L. HDAC2 deregulation in tumorigenesis is causally connected to repression of immune modulation and defense escape. Oncotarget. 2015; 6: 886-901. doi: 10.18632/oncotarget.2816.

51. Seo SK, Hwang CS, Choe TB, Hong SI, Yi JY, Hwang SG, Lee HG, Oh ST, Lee YH, Park IC. Selective inhibition of histone deacetylase 2 induces p53-dependent survivin downregulation through MDM2 proteasomal degradation. Oncotarget. 2015; 6 : 26528-26540. doi: 10.18632/oncotarget.3100.

52. Wilson AJ, Lalani AS, Wass E, Saskowski J, Khabele D. Romidepsin (FK228) combined with cisplatin stimulates DNA damage-induced cell death in ovarian cancer. Gyn Oncol. 2012; 127: 579 - 586.

53. Seiler JA, Conti C, Syed A, Aladjem MI, Pommier Y. The intra-S-phase checkpoint affects both DNA replication initiation and elongation: single-cell and -DNA fiber analyses. Mol Cell Biol. 2007; 27: 5806-5818.

54. Frew AJ, Johnstone RW, Bolden JE. Enhancing the apoptotic and therapeutic effects of HDAC inhibitors. Cancer Lett. 2009; 280: 125-133
55. Haberland M, Montgomery RL, Olson EN. The many roles of histone deacetylases in development and physiology: implications for disease and therapy. Nat Rev Genet. 2009; 10:32-42.

56. Wilson AJ, Holson E, Wagner F, Zhang YL, Fass DM, Haggarty SJ. The DNA damage mark $\mathrm{pH} 2 \mathrm{AX}$ differentiates the cytotoxic effects of small molecule HDAC inhibitors in ovarian cancer cells. Cancer Biol Ther. 2011; 12: 484 - 493.

57. Lu C, Zhu F, Cho YY, Tang F, Zykova T, Ma WY, Bode AM, Dong Z. Cell apoptosis: requirement of $\mathrm{H} 2 \mathrm{AX}$ in DNA ladder formation, but not for the activation of caspase-3. Molecular Cell. 2006; 23: 121-132.

58. Huang X, Kurose A, Tanaka T, Traganos F, Dai W, Darzynkiewicz Z. Sequential phosphorylation of Ser-10 on histone $\mathrm{H} 3$ and ser-139 on histone H2AX and ATM activation during premature chromosome condensation: relationship to cell-cycle phase and apoptosis. Cytometry. 2006; 69: 222-229.

59. Jeggo PA, Geuting V, Löbrich M. The role of homologous recombination in radiation-induced double-strand break repair. Radiother Oncol. 2011; 101: 7-12.

60. Sakai W, Swisher EM, Jacquemont C, Chandramohan KV, Couch FJ, Langdon SP, Wurz K, Higgins J, Villegas E, Taniguchi T. Functional restoration of BRCA2 protein by secondary BRCA2 mutations in BRCA2-mutated ovarian carcinoma. Cancer Res. 2009; 69: 6381-6386.

61. Miller KM, Maas NL, Toczyski DP. Taking it off: regulation of H3 K56 acetylation by Hst 3 and Hst4. Cell Cycle. 2006; 5: 2561 - 2565.

62. Murr R, Loizou JI, Yang YG, Cuenin C, Li H, Wang ZQ, Herceg Z. Histone acetylation by Trrap-Tip60 modulates loading of repair proteins and repair of DNA double-strand breaks. Nat Cell Biol. 2006; 8: 91-99.

63. Xhemalce B, Miller KM, Driscoll R, Masumoto H, Jackson SP, Kouzarides T, Verreault A, Arcangioli B. Regulation of histone H3 lysine 56 acetylation in Schizosaccharomyces pombe. J Biol Chem. 2007 18; 282: 15040 - 15047.

64. Miller KM, Tjeertes JV, Coates J, Legube G, Polo SE, Britton S, Jackson SP. Human HDAC1 and HDAC2 function in the DNA-damage response to promote DNA nonhomologous end-joining. Nat Struct Mol Biol. 2010; 17: 1144-1151.

65. Wu L, Wang Y, Liu Y, Yu S, Xie H, Shi X, Qin S, Ma F, Tan TZ, Thiery JP, Chen L. A central role for TRPS1 in the control of cell cycle and cancer development. Oncotarget. 2014 ; 5: 7677-7690. doi: 10.18632/oncotarget.2291.

66. Faratian D, Zweemer AJ, Nagumo Y, Sims AH, Muir M, Dodds M, Mullen P, Um I, Kay C, Hasmann M, Harrison DJ, Langdon SP. Trastuzumab and pertuzumab produce changes in morphology and oestrogen receptor signalling in ovarian cancer xenografts revealing new treatment strategies. Clin Cancer Res. 2011; 17, 4451-4461.

67. Alvarenga AV, Pereira WC, Infantosi AF, Azevedo CM. Complexity curve and grey level co-occurrence matrix in 
the texture evaluation of breast tumor on ultrasound images. Medical physics. 2007; 34: 379-387.

68. Haralick RM. Statistical and structural approaches to texture. Proc. IEEE. 1979; 67: 786-804

69. Sacile R, Montaldo E, Ruggiero C, Nieburgs HE, Nicolò G. A decision support system to detect morphologic changes of chromatin arrangement in normal-appearing cells. IEEE Trans Nanobioscience. 2003; 2: 118-123.

70. Camp RL, Chung GG. Automated subcellular localization and quantification of protein expression in tissue microarrays. Nat Med. 2002; 8: 1323-1327. 